\title{
Determination of residual sodium content in silver and silver oxide composite materials
}

\author{
R. Vivier ${ }^{*}$, L. Muhr ${ }^{2}$, H. Muhr ${ }^{2}$ and E. Plasari² \\ ${ }^{1}$ Schneider Electric SA, 33 bis, avenue du Maréchal Joffre, 92002 Nanterre Cedex, France \\ ${ }^{2}$ LSGC/CNRS - ENSIC/INPL, 1, rue Grandville, BP 451, 54001 Nancy Cedex, France
}

\begin{abstract}
The presence of silver ions deforms strongly the linear response for sodium determination by flame spectrophotometry. Relative errors up to $60 \%$ can be obtained if linear instead of non-linear relations are used. A correction function is defined and a method based on mathematical considerations is proposed for elimination of interference in determination of sodium concentration.

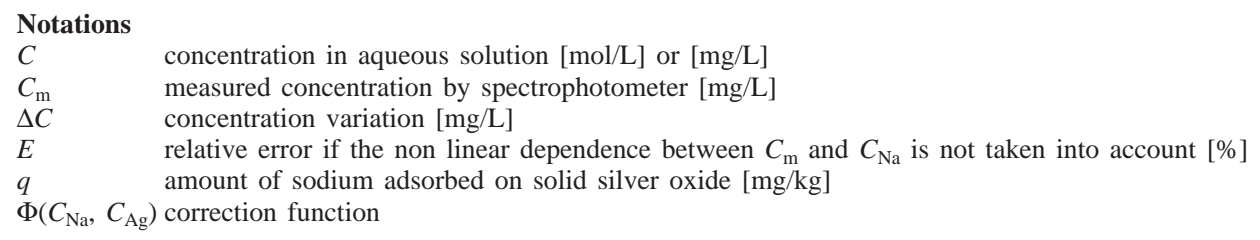

Keywords. Sodium content - impurity - silver - metal oxide - matrix effect - flame spectrophotometer.

\section{Introduction}

Silver-metal oxide materials are used in several electrical switchers. Many composite materials like Ag-CdO, Ag$\mathrm{SnO}_{2}, \mathrm{Ag}-\mathrm{NiO}, \mathrm{Ag}-\mathrm{CuO} \ldots$ have been used or are being developed as contact materials [1]. The mechanical, electrical and metallurgical properties of such composite materials are mainly governed by the powder processing technique as these properties lay on the homogeneity of the metal oxide phase dispersion in silver. As a result, there are several powder processing routes [1]. The present investigation starts with the precipitation of silver oxide in the presence of a metal oxide phase by mixing silver nitrate and sodium hydroxide solutions. The solid obtained must be carefully washed with distilled water in order to eliminate the sodium nitrate, but some quantity of sodium remains in the material. Because of a single electron on the valency layer and a low sublimation point, sodium tends to prime or reprime arcing under electric field, damaging the material in electrical devices. So, it is important to reduce the sodium content to a sufficiently low level in order to obtain a high quality product. Furthermore, care must be taken in the determination of very small quantity of sodium, more particularly in the materials containing large amounts of silver or silver oxide.

This paper deals with a procedure to determine the residual sodium content in silver oxide-metal oxide (intermediary product) and silver-metal oxide composites. In addition, an equilibrium curve showing the evolution of sodium content in the intermediary product as a function of sodium concentration in the washing liquid is given. So the residual sodium content in the solid may be subsequently deduced by measurement of the sodium concentration in the corresponding washing liquid. The influence of both silver and sodium contents on the procedure is also investigated.

\section{Theoretical background of the method}

The principle of the flame spectrophotometer measurement can be briefly reminded: when metal ions in solution are aspirated into a low temperature flame in an aerosol form, the electrons of the atoms are excited to higher energy states. When these electrons return to the ground state, they release the excitation energy and a discrete wavelength of visible light is emitted. This light wavelength can be isolated from other wavelengths by an appropriate optical filter and the amount of light emitted can be quantified with a suitable photometer. For low concentrations of metal ions, the amount of light emitted is usually proportional to the number of atoms in the flame, that is to say to the analyte concentration in solution.

It must be noted here that for a given sodium concentration, the amount of light emitted is also dependent on the matrix composition, hence on the silver concentration in the medium considered.

Two methods can overcome this difficulty: the use of standards with the same silver concentration, or the use of commercial standards with a recovery method. The first

*Correspondence and reprints.

Received December 6, 1999; revised June 15, 2000; accepted July 4, 2000. 


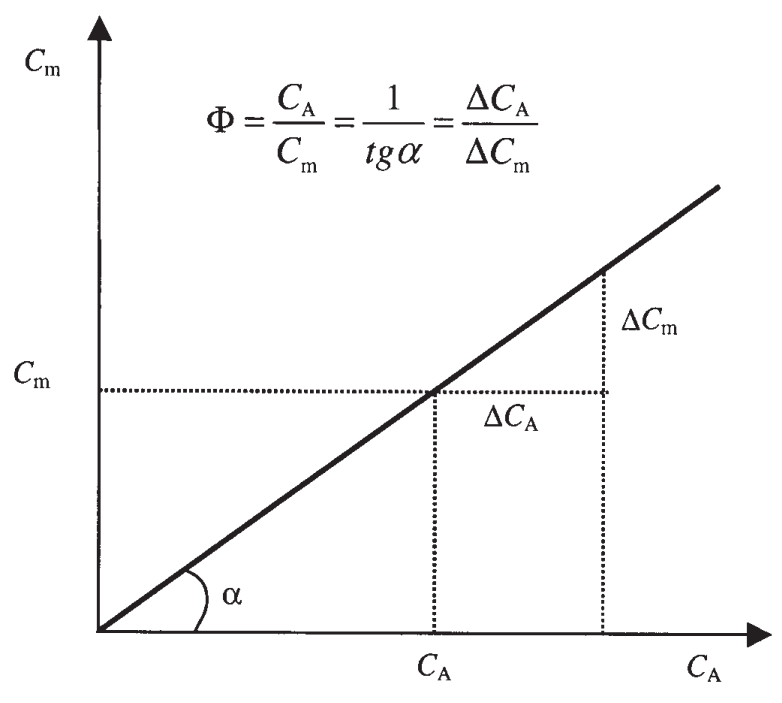

a)

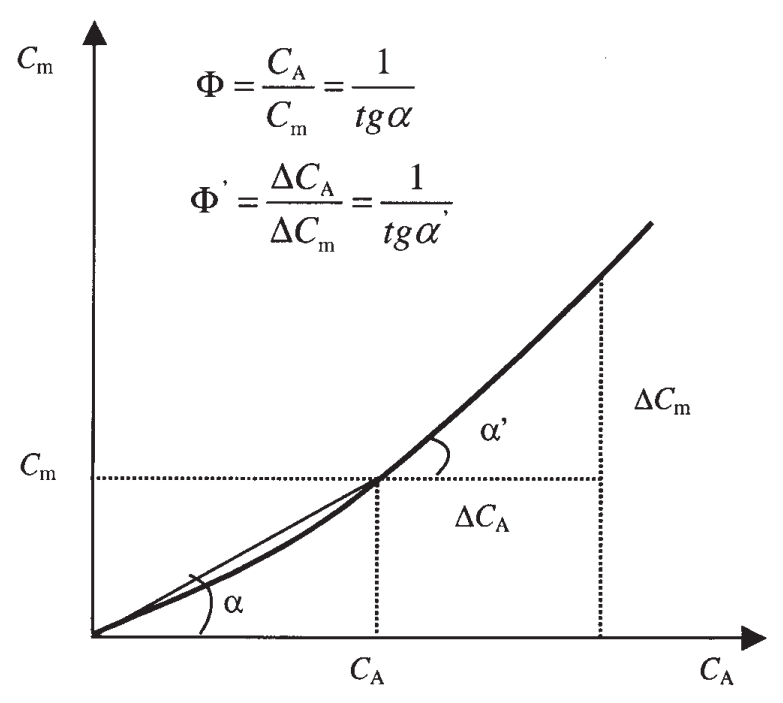

b)

Figure 1. Illustration of the function $\Phi$ variation with $C_{A}$ concentration, in the case of a solution having a constant $C_{B}$ concentration: a) linear case, b) non-linear case.

method is time consuming because every sample corresponds to a different silver content. For such a reason, the second method is preferred. It can be noted that some authors try to overcome this difficulty by the addition of aluminum nitrate buffer to reduce the interferences [2] or by the addition of diluted phosphoric acid to attenuate the emission sensibility of $\mathrm{Na}_{2} \mathrm{O}$ [3]. However, this kind of method increases the risk to pollute the medium.

In the recovery method, the measurement of the concentration of an element $\mathrm{A}$ in a solution containing an element $\mathrm{B}$ able to generate a matrix effect can be corrected using a function $\Phi$ :

$$
C_{\mathrm{A}}=\Phi\left(C_{\mathrm{A}}, C_{\mathrm{B}}\right) C_{\mathrm{m}}
$$

In equation (1), $C_{\mathrm{m}}$ is the sodium concentration measured. It corresponds to a silver free calibration line with the data of the sodium standards diluted in pure water. At first view, it seems easier to work directly with the ratio of light intensity values I, but actually, the calibration line is unsteady because of flame temperature and sample flow variations. Therefore, standards having a concentration close to the one of the sample are used before each measurement. The I signal of this sample is converted to the concentration value, which does not suffer from variations of the measurement conditions. The principle of the method is described on figure 1 in the case of species used in the present study: $C_{\mathrm{A}}$ is the concentration of the element to be measured (i.e.: sodium), whereas $C_{\mathrm{B}}$ is the concentration of the element which generates a matrix effect (i.e.: silver).

Sometimes, the correction function $\Phi$ depends only on $C_{\mathrm{B}}, \Phi=\Phi\left(C_{\mathrm{B}}\right)$. Therefore, for a fixed concentration of the element $\mathrm{B}$, the correction function is constant and a linear dependence exists between $C_{\mathrm{A}}$ and $C_{\mathrm{m}}$ (see equation 1). The real concentration $C_{\mathrm{A}}$ of the element $\mathrm{A}$ in the solution containing high amounts of the element $\mathrm{B}$ is determined in two steps. First, the concentration $C_{\mathrm{m}}$ is measured using the $\mathrm{B}$ free calibration line. After that, a known quantity of the element $\mathrm{A}$ is added to the sample solution allowing to calculate $\Delta C_{\mathrm{A}}$. A second measurement using the $\mathrm{B}$ free calibration line gives $\Delta C_{\mathrm{m}}$. Thus the correction function $\Phi$ is easily obtained according to the formula $\Phi=\Delta C_{\mathrm{A}} / \Delta C_{\mathrm{m}}$ (see Fig. 1.a), and the real concentration of the element $\mathrm{A}$ is obtained through the equation (1).

In general, the correction function depends also on $C_{\mathrm{A}}$, $\Phi=\Phi\left(C_{\mathrm{A}}, C_{\mathrm{B}}\right)$. Thus, for a given concentration of the element $\mathrm{B}$, the dependence of $C_{\mathrm{A}}$ upon $C_{\mathrm{m}}$ is not linear. Figure 1.b shows well that the previous method for the determination of $C_{\mathrm{A}}$ cannot be used, because $C_{\mathrm{A}} / C_{\mathrm{m}} \neq \Delta C_{\mathrm{A}} / \Delta C_{m}\left(C_{\mathrm{A}} / C_{\mathrm{m}}=\Phi\right.$ and $\left.\Delta C_{\mathrm{A}} / \Delta C_{m}=\Phi^{\prime}\right)$. Experiments have shown that the presence of silver attenuates the light intensity ( $\Phi$ is greater than 1) and reinforces the non-linearity between $C_{\mathrm{A}}$ and $C_{\mathrm{m}}$ (see Fig. 2). For this reason, a special way of treating the experimental data is required to obtain the real value of $\mathrm{A}$ concentration from known $\Delta C_{\mathrm{A}}$ and measured $\Delta C_{\mathrm{m}}$.

The way the correction can be done, on a practical point of view, depends on the sensibility of $\Phi$ towards $C_{\mathrm{A}}$.

Hence, for a given concentration of $\mathrm{B}, C_{\mathrm{B}}$ :

$$
\frac{\partial C_{\mathrm{A}}}{\partial C_{\mathrm{m}}}=\Phi\left(C_{\mathrm{A}}, C_{\mathrm{B}}\right)+C_{\mathrm{m}} \frac{\partial \Phi}{\partial C_{\mathrm{m}}}
$$




\section{Original articles}

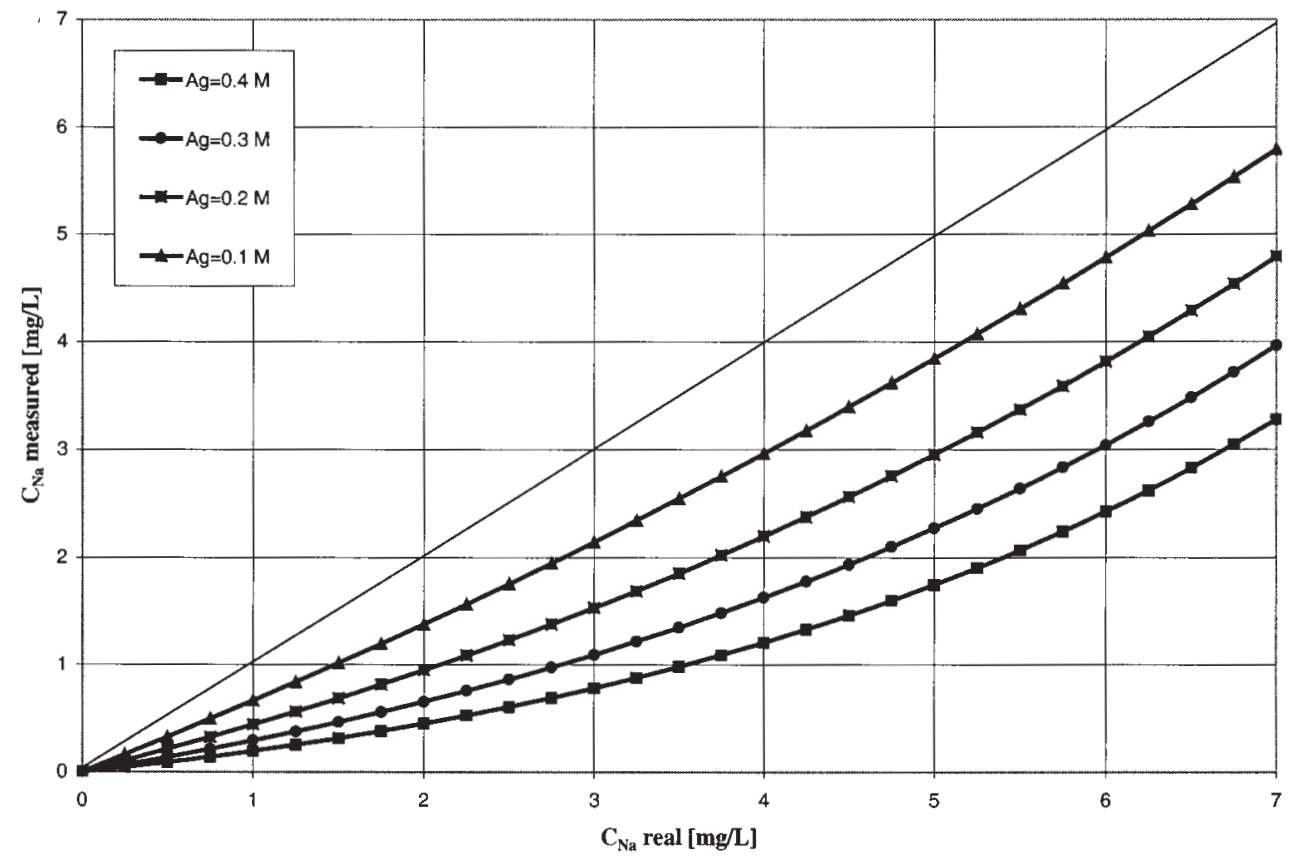

Figure 2. Non-linear effect of silver on sodium spectrophotometer measurement.

$$
\frac{\partial C_{\mathrm{A}}}{\partial C_{\mathrm{m}}}=\Phi\left(C_{\mathrm{A}}, C_{\mathrm{B}}\right)+C_{\mathrm{m}} \frac{\partial \Phi}{\partial C_{\mathrm{A}}} \frac{\partial C_{\mathrm{A}}}{\partial C_{\mathrm{m}}}
$$

therefore:

$$
\Phi\left(C_{\mathrm{A}}, C_{\mathrm{B}}\right)=\frac{\partial C_{\mathrm{A}}}{\partial C_{\mathrm{m}}}\left(1-C_{\mathrm{m}} \frac{\partial \Phi}{\partial C_{\mathrm{A}}}\right)
$$

The variation of the measured concentration when $C_{\mathrm{A}}$ varies is experimentally evaluated by adding a known amount of $\mathrm{A}$ in the solution, noted $\Delta C_{\mathrm{A}}$, and measuring the corresponding increase $\Delta C_{\mathrm{m}}$.

Then, according to equation (4), we can write:

$$
\Phi\left(C_{\mathrm{A}}, C_{\mathrm{B}}\right) \approx \frac{\Delta C_{\mathrm{A}}}{\Delta C_{\mathrm{m}}}\left(1-C_{\mathrm{m}} \frac{\partial \Phi}{\partial C_{\mathrm{A}}}\right)
$$

Two situations can be considered:

1. If the function $\Phi$ is not sensible to A concentration variations, $\frac{\partial \Phi}{\partial C_{\mathrm{A}}} \approx 0$. It is usually the situation when $C_{\mathrm{A}}$ is small enough: then measurements are carried out in what is classically called the "linear range".

$$
\Phi \approx \frac{\Delta C_{\mathrm{A}}}{\Delta C_{\mathrm{m}}}
$$

The results obtained can then be easily corrected:

$$
C_{\mathrm{A}} \approx \frac{\Delta C_{\mathrm{A}}}{\Delta C_{\mathrm{m}}} C_{\mathrm{m}}
$$

2. If the function $\Phi$ is sensible to A concentration variations, $\frac{\partial \Phi}{\partial C_{\mathrm{A}}}$ cannot be neglected any more. In this situation, preliminary experiments must be carried out using synthetic solutions of known concentrations of $\mathrm{A}$ and $\mathrm{B}$ in order to determine $\Phi\left(C_{\mathrm{A}}, C_{\mathrm{B}}\right)$.

Two cases can then be encountered when A concentration must be evaluated :

\section{B concentration is known}

In this case, $C_{\mathrm{A}}$ is solution of the implicit equation (1), which can be solved by successive iterations using $C_{\mathrm{A} 0}=C_{\mathrm{m}}$ as initial value.

B concentration is unknown, which is the case usually encountered

The determination of $\Phi$ is based on equation (5). The mathematical method used depends then on $\Phi$ expression. This problem will be treated later in the case of sodium (A) and silver (B).

\section{Experimental}

\section{Equipment and operating procedure}

A 410C CORNING flame spectrophotometer is used to measure the sodium content. The calibration line is determined through $0,1,5$ and $10 \mathrm{mg} / \mathrm{l}$ sodium standards. Preliminary measurements are carried out with synthetic solutions of known concentration of sodium and silver in order to determine $\Phi\left(C_{\mathrm{Na}}, C_{\mathrm{Ag}}\right)$. 


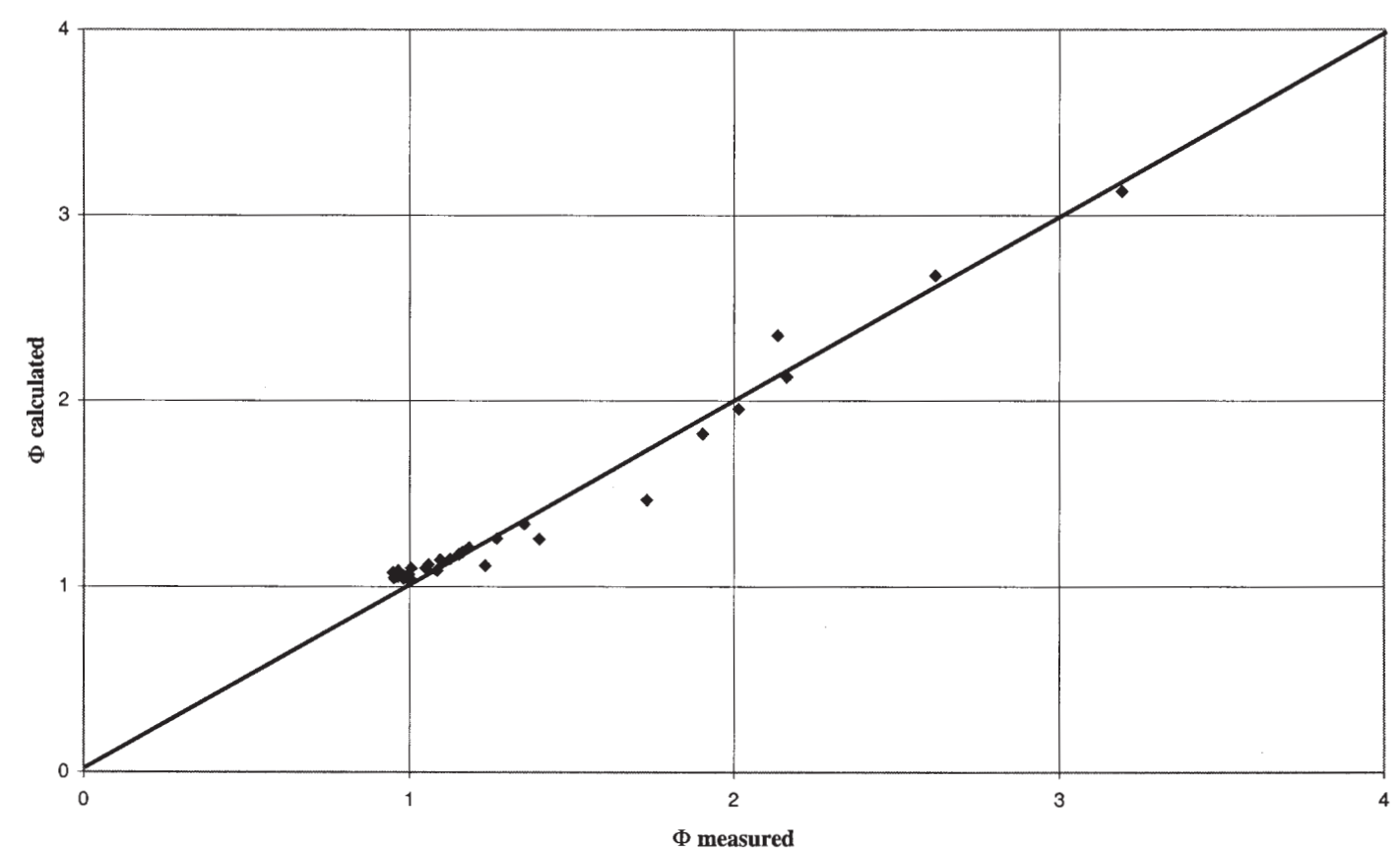

Figure 3. Experimental validation of $\Phi$ correlation.
Silver and silver oxide materials, about 5 to $10 \mathrm{~g}$, can be rendered soluble by the nitric acid attack $\left(68 \% \mathrm{HNO}_{3}\right)$ [4]. As the sodium content is very low, Teflon instruments and containers must be used because glass vessel would release sodium ions. It is actually admitted that the main cause of error for sodium determination is the contamination by other sodium containing sources [5,6] (glass-ware, reagent purity, dilution preceding the measurement) if precautions are not taken. The attack can be completed through microwave heating. The microwave oven used must be installed under a hood equipped with a system enabling the evacuation of toxic nitrous fumes, especially if the method is used for metallic silver material. In the present work, the other metal oxide remains insoluble after this attack. Then, the solution is filtered on $0.45 \mu \mathrm{m}$ Millipore filter made of cellulose nitrate. The residue must be washed several times carefully with hot water, as oxides are well known to adsorb ions.

The whole liquid is completed to known volume with distilled water. The sodium concentration is measured. Then 25 to $100 \mu \mathrm{L}$ of sodium nitrate standard $1000 \pm 1 \mathrm{mg} / \mathrm{L}$ is added to $100 \mathrm{~cm}^{3}$ of the sample solution and the sodium concentration increase is measured.

\section{Determination of $\Phi\left(C_{\mathrm{Na}}, C_{\mathrm{Ag}}\right)$}

Synthetic solutions of silver and sodium nitrate are prepared and sodium concentration is measured using the standards described previously. The measured concentration to the real concentration ratio can be quite different to one: the presence of silver has a great effect on sodium emission measurement as it reabsorbs part of the energy released. In our case, the matrix effect sensibly attenuates the sodium emission as it is usually observed. Nevertheless, enhancement has already been reported in literature. Agudelo et al. [7] found for instance that the presence of lithium metaborate enhances sodium emission, whereas the presence of iron, aluminum and potassium does not affect it.

From figure 2, it can be observed that, for a given silver concentration, the ratio $C_{\mathrm{Na}} / C_{\mathrm{m}}$ increases when the sodium concentration increases. In addition, the combination of low sodium content and silver reach matrix is a critical scenario, while low silver content has almost no influence on sodium concentration measurement.

Using these experimental data, an expression for the function $\Phi\left(C_{\mathrm{Na}}, C_{\mathrm{Ag}}\right)$ is searched.

The relation :

$$
\Phi\left(C_{\mathrm{Na}}, C_{\mathrm{Ag}}\right)=\exp \left(a C_{\mathrm{Ag}}\left(1+b C_{\mathrm{Na}}\right)\right)
$$

with $a=4.47$ and $b=-8.2310^{-2}$ when $C_{\mathrm{Na}}$ is given in $\mathrm{mg} / \mathrm{L}$ (or ppm) and $C_{\mathrm{Ag}}$ in $\mathrm{mol} / \mathrm{L}$ enables to fit the previous results with a mean error of $5 \%$ in the range $C_{\mathrm{Na}}<7 \mathrm{ppm}$, $C_{\mathrm{Ag}}<0.5 \mathrm{~mol} / \mathrm{L}$ (see Fig. 3 ).

\section{Results and discussion}

From equation (8), we can easily obtain :

$$
\frac{\partial \Phi}{\partial C_{\mathrm{NA}}}=a b C_{\mathrm{Ag}} \exp \left(a C_{\mathrm{Ag}}\left(1+b C_{\mathrm{Na}}\right)\right)=a b C_{\mathrm{Ag}} \Phi
$$

equation (8) gives also :

$$
C_{\mathrm{Ag}}=\frac{\ln \Phi}{a\left(1+b C_{\mathrm{Na}}\right)}
$$




\section{Original articles}

The substitution of equation (10) to equation (9) yields :

$$
\frac{\partial \Phi}{\partial C_{\mathrm{Na}}}=\frac{b \Phi \ln \Phi}{1+b C_{\mathrm{Na}}}=\frac{b \Phi \ln \Phi}{1+b \Phi C_{\mathrm{m}}}
$$

thus equation (5) can be transformed to:

$$
\Phi=\frac{\Delta C_{\mathrm{Na}}}{\Delta C_{\mathrm{m}}}\left(1-\frac{b \Phi C_{\mathrm{m}}}{1+b \Phi C_{\mathrm{m}}} \ln \Phi\right)
$$

Equation (12) is the basic equation for the determination of sodium concentration when silver concentration is not known. Indeed, with experimental values $\Delta C_{\mathrm{Na}}, \Delta C_{\mathrm{m}}$ and $C_{\mathrm{m}}$, equation (12) represents an implicit equation, from which $\Phi$ can be easily obtained by an iterative method using as initial value $\Phi=1$. Afterwards, knowing $C_{\mathrm{m}}$ and $\Phi$, the real sodium concentration can be calculated by equation (1). It is interesting to evaluate the error of sodium concentration determination if the non linear dependence between $C_{\mathrm{m}}$ and $C_{\mathrm{Na}}$ is not taken into account. The relative error is directly obtained from the comparison of equation (5) with equation (6):

$$
E=\frac{\Phi_{\text {non-linear }}-\Phi_{\text {linear }}}{\Phi_{\text {non-linear }}}=\frac{-C_{\mathrm{m}} \frac{\partial \Phi}{\partial C_{\mathrm{Na}}}}{1-C_{\mathrm{m}} \frac{\partial \Phi}{\partial C_{\mathrm{Na}}}}=\frac{-a b C_{\mathrm{Na}} C_{\mathrm{Ag}}}{1-a b C_{\mathrm{Na}} C_{\mathrm{Ag}}}
$$

The upper values of sodium and silver concentrations in this study are $C_{\mathrm{Na}}=7 \mathrm{mg} / \mathrm{L}$ and $C_{\mathrm{Ag}}=0.5 \mathrm{~mol} / \mathrm{L}$. With these values, equation (13) gives a relative maximum error $\mathrm{err}_{\max }=0.56(56 \%)$, which proves the necessity for the use of the procedure described above for the determination of sodium traces in high silver content materials.

\section{Example of application}

The procedure exposed previously is applied in the case of artificial samples having known sodium concentrations. The results obtained are given in table I.

Going out from results presented in table I, it can be observed that when silver concentration increases, hence, when the non-linearity increases, the precision of the results decreases a bit, reaching around $12 \%$ in the worst cases. Nevertheless, the error corresponding to the non linear dependence between $C_{\mathrm{m}}$ and $C_{\mathrm{Na}}$ is much more higher than this one. Indeed, the last column of table I shows the respective error $\mathrm{E}$ calculated from $\frac{\Delta C_{\mathrm{Na}}}{\Delta C_{\mathrm{m}}}$ and $\Phi$ values according to the formula:

$$
E=\frac{\Phi_{\text {non-linear }}-\Phi_{\text {linear }}}{\Phi_{\text {non-linear }}} \approx \frac{\Phi-\frac{\Delta C_{\mathrm{Na}}}{\Delta C_{\mathrm{m}}}}{\Phi}
$$

In the case of very low sodium concentration and low silver concentration, the error $\mathrm{E}$ is of the same order of magnitude than the error of sodium determination, because in this range the dependence between $C_{\mathrm{m}}$ and $C_{\mathrm{Na}}$ is almost linear. On the contrary, for higher sodium and silver concentrations, the non linearity beween $C_{\mathrm{m}}$ and $C_{\mathrm{Na}}$ becomes more and more pronounced, giving an E-error 2 to 10 times higher than the error of sodium concentration determination. Thus, the comparison of two last columns shows clearly the advantage of the present method which takes into account the non linearity between $C_{\mathrm{m}}$ and $C_{\mathrm{Na}}$.

It must also be noted that when equation (12) is solved, two solutions are numerically obtained: a choice must therefore be realized, considering the "physically" possible solution. However, in a few cases it appears difficult to choose: thus, it is necessary to have a rough estimate of silver concentration.

\section{Determination of the washing equilibrium curve for the silver oxide composite}

The previous method has been used to determine the washing equilibrium curve for solid silver oxide-metal oxide composite obtained by the precipitation route and contaminated with sodium nitrate. In the present case, solid silver nitrate is dissolved in distilled water and after that, the metal oxide is suspended in the silver nitrate solution. A sodium hydroxide solution is poured in the previous suspension under vigorous agitation. Precipitation of silver oxide occurs instantaneously. Afterwards, a separation allows to collect apart liquid and solid. The solid phase is washed several times with a constant distilled water volume. At each wash-

\begin{tabular}{|c|c|c|c|c|c|c|c|c|}
\hline Known concentrations of samples & $\begin{array}{c}C_{\mathrm{m}} \\
{[\mathrm{mg} / \mathrm{L}]}\end{array}$ & $\begin{array}{c}\Delta C_{\mathrm{Na}} \\
{[\mathrm{mg} / \mathrm{L}]}\end{array}$ & $\begin{array}{c}\Delta C_{\mathrm{m}} \\
{[\mathrm{mg} / \mathrm{L}]}\end{array}$ & $\frac{\Delta C_{\mathrm{Na}}}{\Delta C_{\mathrm{m}}}$ & $\Phi$ & $\begin{array}{c}C_{\mathrm{Na}} \text { obtained } \\
{[\mathrm{mg} / \mathrm{L}]}\end{array}$ & $\underset{\%}{\text { error }}$ & $\begin{array}{l}E \\
\%\end{array}$ \\
\hline$C_{\mathrm{Ag}}=0.1 \mathrm{~mol} / \mathrm{L} \quad C_{\mathrm{Na}}=\mathbf{0 . 5} \mathrm{mg} / \mathrm{L}$ & 0.33 & 0.25 & 0.17 & 1.47 & 1.50 & 0.49 & 2.0 & 2.0 \\
\hline$C_{\mathrm{Ag}}=0.1 \mathrm{~mol} / \mathrm{L} \quad C_{\mathrm{Na}}=3.5 \mathrm{mg} / \mathrm{L}$ & 2.55 & 0.25 & 0.21 & 1.19 & 1.31 & 3.34 & 4.6 & 9.2 \\
\hline$C_{\mathrm{Ag}}=0.1 \mathrm{~mol} / \mathrm{L} \quad C_{\mathrm{Na}}=6.5 \mathrm{mg} / \mathrm{L}$ & 5.28 & 0.25 & 0.25 & 1.00 & 1.21 & 6.39 & 1.7 & 17.4 \\
\hline$C_{\mathrm{Ag}}=0.2 \mathrm{~mol} / \mathrm{L} \quad C_{\mathrm{Na}}=3.5 \mathrm{mg} / \mathrm{L}$ & 1.85 & 0.25 & 0.17 & 1.47 & 1.79 & 3.31 & 5.4 & 17.9 \\
\hline$C_{\mathrm{Ag}}=0.3 \mathrm{~mol} / \mathrm{L} \quad C_{\mathrm{Na}}=\mathbf{3 . 5} \mathrm{mg} / \mathrm{L}$ & 1.35 & 0.25 & 0.14 & 1.79 & 2.29 & 3.09 & 11.7 & 21.8 \\
\hline$C_{\mathrm{Ag}}=0.4 \mathrm{~mol} / \mathrm{L} \quad C_{\mathrm{Na}}=3.5 \mathrm{mg} / \mathrm{L}$ & 0.98 & 0.25 & 0.11 & 2.27 & 3.18 & 3.12 & 10.9 & 28.6 \\
\hline
\end{tabular}
ing step, after the equilibrium state is reached, solid and liquid phases are sampled and kept for analysis.

Table I. Example of sodium measurement with the recovery method. 


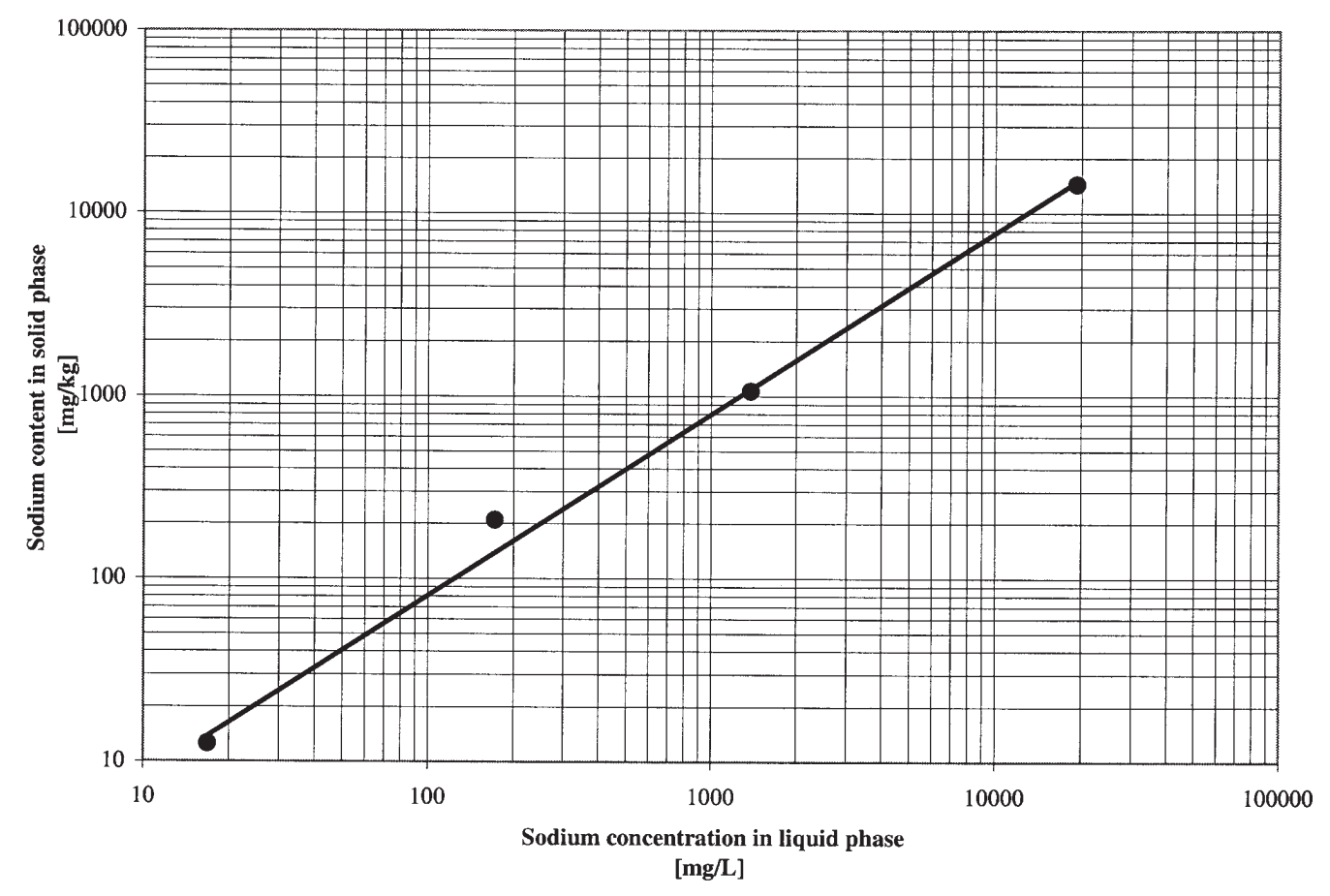

Figure 4. Sodium washing equilibrium curve of silver oxide.
The washing equilibrium curve at $20^{\circ} \mathrm{C}$ is represented in figure 4 . The adsorption of sodium ions on silver oxide can be characterized by the Langmuir equation, which is reduced to a linear relationship for very low sodium concentration in the liquid phase. Indeed, the log-log plot of sodium content in the solid phase as a function of sodium concentration in the liquid phase shown on figure 4 has a slope equal to one. In our case, the equilibrium washing curve is given with good agreement by: $\mathrm{q}=7.455 \cdot 10^{-1} C_{\mathrm{Na}}, C_{\mathrm{Na}}$ being the sodium concentration in the liquid phase (in $\mathrm{mg} / \mathrm{L}$ ) and $\mathrm{q}$ the sodium concentration in the solid phase (in $\mathrm{mg} / \mathrm{kg}$ or ppm).

It is technically easier to determine sodium content in the liquid phase rather than in the solid one. Thus for industrial processes, such a curve allows to know rapidly the residual sodium content in the silver-metal oxide composite directly from the sodium concentration in the washing liquid. The industrial washing procedure may be better controlled by this way.

\section{Conclusion}

The presence of silver in a sample has a great influence on sodium emission measured by flame spectrophotometry. The light intensity is attenuated and becomes highly non-linear towards the sodium concentration. In this situation, the classical techniques of residual sodium content determination take well into account the light emission attenuation by the addition in the sample of a known amount of sodium. Nevertheless, high relative errors can be obtained, because these techniques use linear instead of non-linear relations.
The definition of a correction function for the characterisation of the non-linearity range is shown to be very useful for the quantification of sodium traces in silver composite materials. In spite of intermediary mathematical operations of a certain degree of complexity, the final relation is simple and easy to use. The same methodology may be applied in similar cases characterised by high matrix effects.

The interest of the method is illustrated by a special case from silver composite material production. The washing process is a very delicate step influencing the quality of such materials. Using the method presented above, a washing equilibrium curve is obtained allowing the rapid determination of sodium content in intermediary products and ensuring this way the good quality of final composite materials.

\section{References}

1. Joshi, P.B.; Patel, R.H.; Krishnan, P.S.; Gadgeel, V.L.; Kaushik, V.K.; Ramakrishnan, P. Advanced Powder Technol. 1996, 7(2), 121-130.

2. Hermann, I. Silikat. Tech. 1983, 34(9), 274.

3. Demoulian, F. Rev. Mat. Constr. 1964, 157, 568.

4. Charlot, G. In Les méthodes de la chimie analytique - Analyse quantitative minérale; Paris: Masson, 1966.

5. Voinovitch, I.A. In Analyse des sols, roches et ciments, Méthodes choisies, Paris Milan Barcelone Mexico: Masson, 1988.

6. Ramirez-Munoz, J. Inform. Chim. Anal. 1967, 21(3), 97.

7. Agudelo, A.; Garcia-Vargas, M.; Coy-Yll, R. Afinidad. 1983, 40(386), 331. 